\title{
Posterior intraprosthetic dislocation of cervical arthroplasty: illustrative case
}

\author{
Marc Prod'homme, MD, Didier Grasset, MD, and Duccio Boscherini, MD, PhD \\ Neuro Orthopedic Center, Clinic La Source, Lausanne, VD, Switzerland
}

BACKGROUND Cervical disc herniation is a common condition usually treated with anterior cervical discectomy and fusion (ACDF) or, more recently, with cervical disc arthroplasty (CDA). Both treatments offer similar clinical results. However, CDA has been found to offer fewer medium- to long-term complications as well as potential reduction of long-term adjacent disc degeneration.

OBSERVATIONS A 40-year-old man was treated with cervical discectomy and arthroplasty due to a C6-C7 disc herniation with left C7 radiculopathy. After the treatment, his postoperative follow-up appointments were uneventful for 9 months. However, after 9 months, he reported cervical pain and a right $\mathrm{C} 7$ radiculopathy after neck extension. Imaging confirmed a posterior intraprosthetic dislocation, the first case reported to date. The patient was received emergency surgery under neuromonitoring, and the prosthesis was replaced by an ACDF and anterior plate. The insert presented a rupture of the anterior horn. The patient presented no preoperative or postoperative neurological deficit, and his follow-up review revealed no issues.

LESSONS Posterior intraprosthetic dislocation is an extremely rare complication. It may occur with Mobi-C cervical arthroplasty in the case of rupture and oxidation of the polyethylene insert. Spine surgeons should be aware of this potential major complication.

https://thejns.org/doi/abs/10.3171/CASE21500

KEYWORDS cervical arthroplasty; Mobi-C; intraprosthetic dislocation; spinal cord compression

Cervical disc disease is a common condition that may lead to cervical disc herniation and radiculopathy of the upper extremities. ${ }^{1}$ Cervical disc herniation was long treated by anterior cervical discectomy and fusion (ACDF) with good results and long-term efficacy and safety. ${ }^{2}$ Over the last two decades, cervical disc arthroplasty (CDA) has become an alternative treatment of cervical disc disease. Some literature reviews found better medium- to long-term ${ }^{3,4}$ clinical results, reoperation rates, and adjacent segmental disease ${ }^{5-7}$ with CDA. Other reviews showed that CDA was not inferior to ACDF, ${ }^{8}$ and a Cochrane review concluded clinical results in favor of CDA. ${ }^{9}$ However, in the latter, the issues discussed were at such a low sample rate of studies with such small differences obtained, not blinded studies, that a bias potentially existed related to caregiver expectations.

Several trademarked products are available, such as Bryan (Spinal Dynamics Corp. and Medtronic Sofamor Danek), ProDisc-C (DePuy Synthes), Prestige (Medtronic), and Mobi-C (Zimmer-Biomet), with different settings and shapes and a common objective of disc motion preservation. The Mobi-C is a semiconstrained cervical prosthesis containing two chrome-cobalt plates and a mobile polyethylene insert in between. ${ }^{10}$

Complications related to CDA are primarily linked to the anterior cervical approach, such as dysphagia (2-70\%), ${ }^{11}$ recurrent laryngeal nerve compression (3-16.7\%), ${ }^{12,13}$ hematoma (suffocating or epidural, incidence $0.2 \%$ and $0.9 \%$, respectively), ${ }^{14}$ dural tear with pseudomeningocele $(0.5-3 \%),{ }^{15}$ esophageal lesion $(0.4-1.15 \%),{ }^{16,17}$ spinal cord compression $(0.5 \%),{ }^{18}$ vertebral artery injury $(0.4 \%),{ }^{18}$ and exceptional tracheal or thoracic duct lesion. $^{19}$

Postoperative neurological impairment is the most dreaded complication in cervical surgery for patients and surgeons. Some specific complications of CDA are well-known, including anterior bone loss $(41.84 \%)^{20}$ and material subsidence, ${ }^{21}$ infection $(<3.7 \%){ }^{22}$ and heterotopic ossifications (7.7-94.1\%). ${ }^{23-25}$

Herein we describe a case of delayed posterior intraprosthetic dislocation of cervical arthroplasty.

ABBREVIATIONS ACDF = anterior cervical discectomy and fusion; $C D A=$ cervical disc arthroplasty; $M R I=$ magnetic resonance imaging; NRS = numerical rating scale.

INCLUDE WHEN CITING Published December 6, 2021; DOI: 10.3171/CASE21500.

SUBMITTED September 2, 2021. ACCEPTED October 22, 2021

(c) 2021 The authors, CC BY-NC-ND 4.0 (http://creativecommons.org/licenses/by-nc-nd/4.0/). 

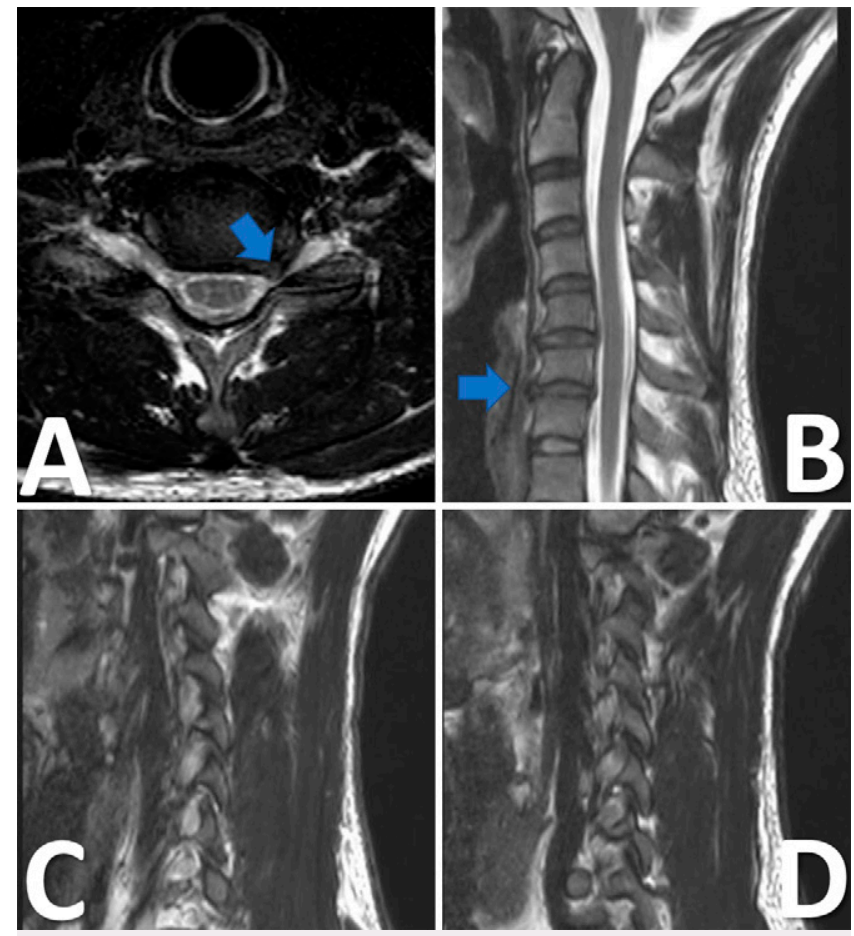

FIG. 1. Preoperative cervical MRI. A: Axial T2-weighted view showing a left C6-C7 disc herniation (arrows). B: Sagittal T2-weighted view showing C6-C7 discopathy. C and D: Right and left sagittal views showing no signs of posterior facet arthritis or instability. We noticed a sigmoid aspect of the cervical spine, considered antalgic.

\section{Illustrative Case}

A 40-year-old male patient (smoker) received surgery by the team at Clinic La Source in Lausanne, Switzerland, because of a left $\mathrm{C} 7$ radiculopathy with motor deficit $\mathrm{M} 4+/ 5$ and pain-resisting medical treatment. Cervical magnetic resonance imaging (MRI) (Fig. 1) found a left C6-C7 disc herniation with $\mathrm{C} 7$ conflict and no sign of posterior facet or instability. The surgery consisted of a right cervicotomy, C6-C7 microdiscectomy, and CDA with a Mobi-C prosthesis (Zimmer-Biomet) (Fig. 2). The patient was discharged from the clinic after 24 hours. The follow-up review showed adequate wound healing, complete resolution of motor deficit, and radicular pain recovery without any complication.

After 9 months, the patient presented to the office after feeling neck pain for 5 days and right $\mathrm{C} 7$ radicular pain after neck extension. The pain was immediately unbearable (numerical rating scale [NRS] of 8/10), with transient right complete motor deficit of the lower limb in a few minutes, so he went to the emergency department in another location (Yverdon-les-Bains). Radiographs were obtained (Fig. 2) and were considered normal. The patient was discharged with analgesics, and he was recommended to consult his surgeon.

Clinical examination showed a NRS 10/10, a Neck Disability Index of $82 \%$, and a well-healed scar without any sign of inflammation. Cervical mobilization was painful and limited to $30^{\circ}$ of rotation. The Spurling test was bilaterally positive, triggering a right $\mathrm{C} 7$ radicular pain. There was no motor or sensitive neurological deficit of the upper limbs. Lhermitte and Hoffmann signs were negative.

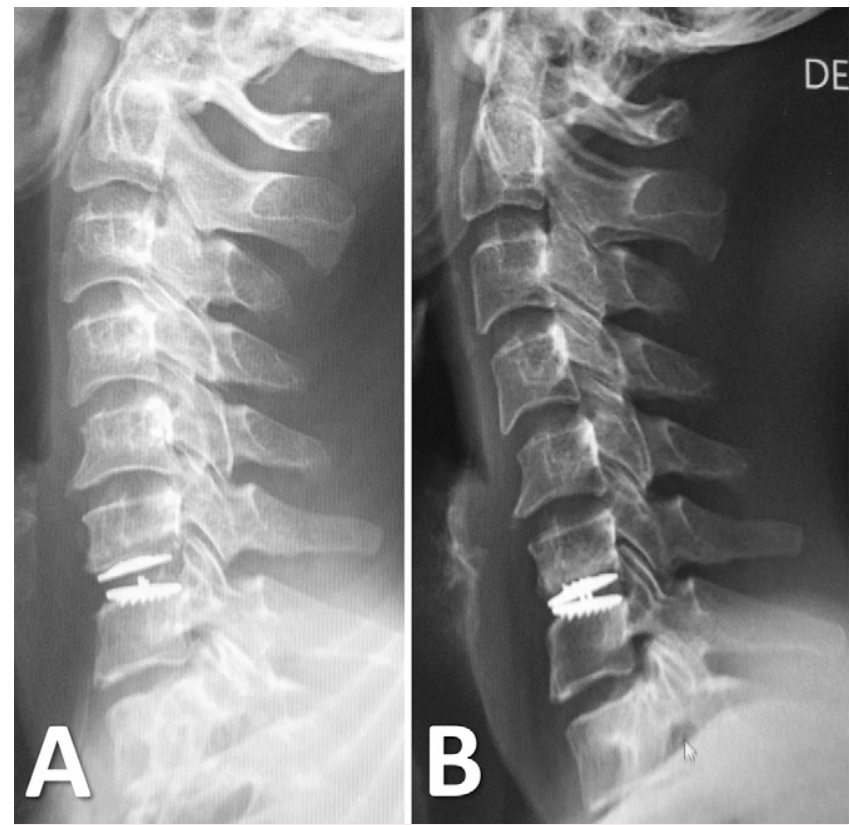

FIG. 2. Lateral radiographs of the cervical spine. A: Immediate postoperative view. B: At 9 months postoperatively; one can notice the loss of height of the intraprosthetic space and anterior closure aspect of the implant, supposing a posterior migration of the polyethylene insert.

Emergency cervical MRI showed a suspicion of intraprosthetic dislocation, so the imaging was completed by computed tomography (Fig. 3), which confirmed the diagnosis.

The patient received emergency surgery the same day under C3-T1 neuromonitoring (NIM Eclipse Surgeon Directed, Medtronic). A left cervicotomy was performed using microscopic magnification. The superior plate of the prosthesis was not adhesive and was easily removed. The mobile part of the prosthesis in polyethylene was posteriorly dislocated and retained by the posterior longitudinal ligament. The anterior horn of the polyethylene insert was ruptured and the global aspect was partially supple, allowing a hook to be inserted within (Fig. 4). The inferior plate of the prosthesis adhered well to the vertebral endplate and was removed using a bone osteotome. A sample of deep membrane was taken for microbiological analysis. A polyether-ether ketone cage was implanted with 1 $\mathrm{cm}^{3}$ of bone substitute in the interbody space. An anterior $\mathrm{C6}-\mathrm{C7}$ plate completed the instrumentation (Fig. 5).

Postoperatively, the patient showed no complications, and the radicular pain was relieved. The patient was discharged from the clinic after 2 days. The sample taken remained negative for microbial culture. The follow-up review was without issue, with adequate wound healing and normal cervical motion after 2 months. The NRS reached $4 / 10$ and Neck Disability Index was $34 \%$.

\section{Discussion}

\section{Observations}

To our knowledge, this is the first case of posterior intraprosthetic dislocation of a CDA reported in the literature so far. Tsermoulas and Bhattathiri ${ }^{26}$ reported the first case of anterior dislocation of a C5-C6 Mobi-C arthroplasty. They found a fixed 


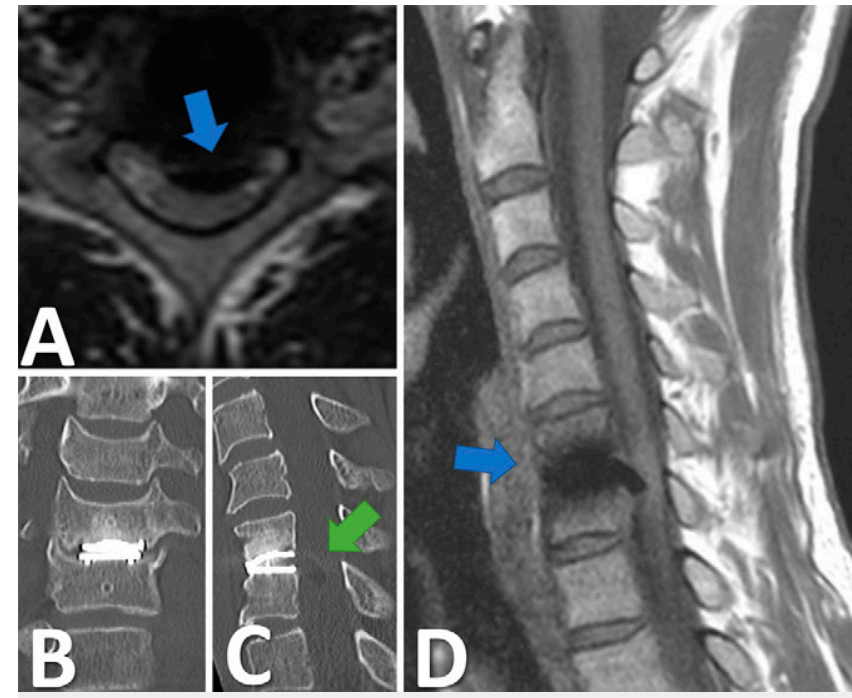

FIG. 3. MRI and computed tomography (CT) of the cervical spine 9 months postoperatively. A: Axial T2-weighted MRI showing artifact of the prosthesis plate and potential anterior spinal cord compression (blue arrow). B: Frontal CT showing anterior contact of the metallic plates, suggesting an absence of the polyethylene insert. C: Sagittal CT demonstrating posterior dislocation of the polyethylene insert (green arrow) and osteolysis around the upper plate. D: Sagittal T1-weighted MRI showing a footprint on the anterior spinal cord (blue arrow) without any signs of myelopathy.

expulsion of the inferior plate and insert from the prosthesis after 8 weeks and performed emergency ACDF with good results. They hypothesized that the lack of prosthesis restraint may have been the main cause of the implant migration. In that case, the 54-yearold female patient had severe vomiting and coughing episodes that may have increased stress on the implant and triggered its dislodgment.

Pelletier et al. described a case of an early anterior intraprosthetic dislocation of a C4-C5 Mobi-C CDA ${ }^{27}$ related to excessive

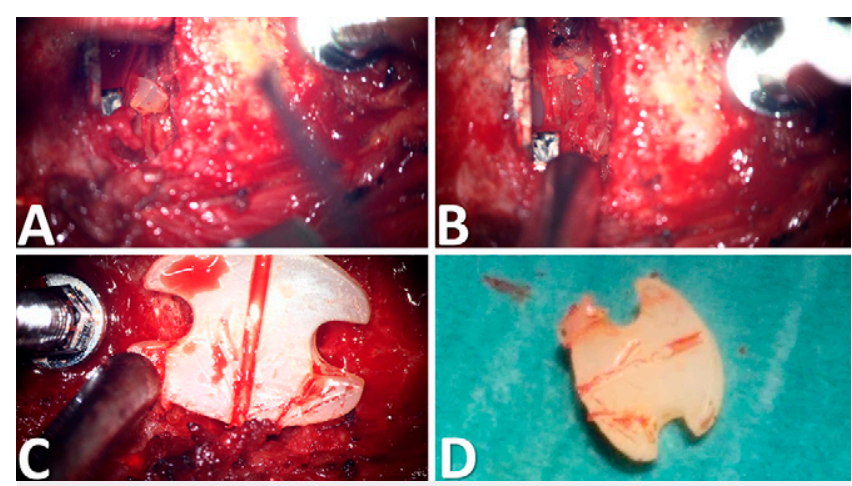

FIG. 4. Intraoperative views of the polyethylene insert. Orientation of $\mathbf{A}$ to $\mathbf{C}$ : left side is caudal; right side is cranial. A: Left anterior rupture fragment. B: Membranes reformed anteriorly to the insert and were sampled for microbiological analysis. C and D: One can notice the ridges on the surface of the implant performed with the microsurgical hook during its extraction and the anterior rupture of the left horn of the insert.

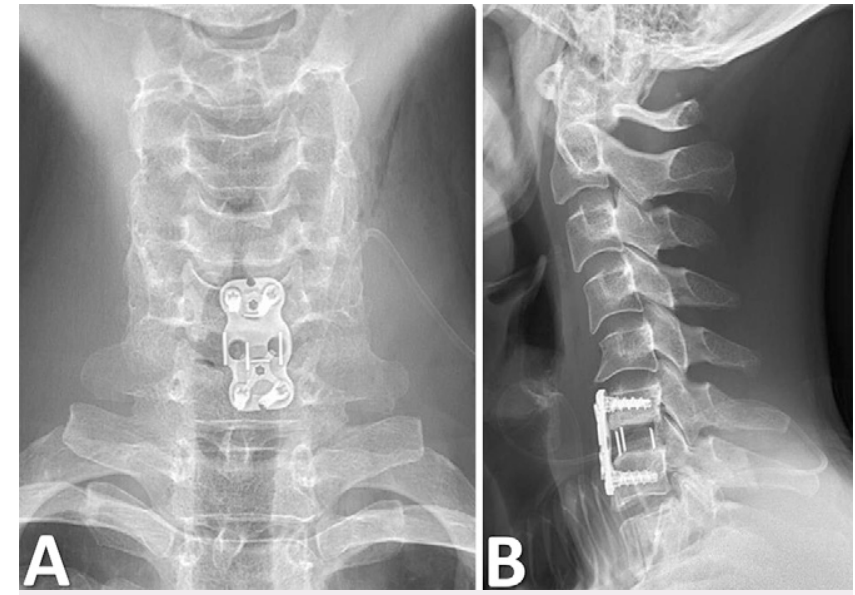

FIG. 5. Postoperative radiographs. A: Anterior-posterior view showing a good position of the plate and the polyether-ether ketone implant for C6-C7 fusion. B: Lateral view showing adequate position of the implants and length of the screws.

motion of the mobile segment adjacent to a two-level C5-C6 and C6-C7 ACDF. The dislocated CDA was removed and ACDF was performed with an anterior plate placed from $\mathrm{C} 4$ to $\mathrm{C} 7$.

Several causes of CDA failure have been discussed, such as inappropriate patient selection, under- or oversized implants, and technical error. ${ }^{28}$ In the current case, the polyethylene insert showed signs of wear and partial rupture. The delay of occurrence and motion of the upper plate suggest a slow mechanism, with plate osteolysis and polyethylene oxidation, which might have been favored by tobacco exposure. ${ }^{29}$ In addition to the lack of restraint of the prosthesis, these mechanisms may have resulted in intraprosthetic dislocation.

\section{Lessons}

Posterior intraprosthetic dislocation is an extremely rare complication that may occur with Mobi-C cervical arthroplasty in cases of rupture and oxidation of the polyethylene insert. Spine surgeons should be aware of this potential major complication. Further studies and investigations are needed to understand the exact causes of CDA failure.

\section{Acknowledgments}

We thank Mrs. Fiona Tweedie for her English language corrections.

\section{References}

1. Wong JJ, Côté P, Quesnele JJ, Stern PJ, Mior SA. The course and prognostic factors of symptomatic cervical disc herniation with radiculopathy: a systematic review of the literature. Spine J. 2014;14(8):1781-1789.

2. Mazas S, Benzakour A, Castelain JE, Damade C, Ghailane S, Gille 0 . Cervical disc herniation: which surgery? Int Orthop. 2019;43(4):761-766.

3. Hu Y, Lv G, Ren S, Johansen D. Mid- to long-term outcomes of cervical disc arthroplasty versus anterior cervical discectomy and fusion for treatment of symptomatic cervical disc disease: a systematic review and meta-analysis of eight prospective randomized controlled trials. PLoS One. 2016;11(2):e0149312. 
4. Makhni MC, Osorio JA, Park PJ, Lombardi JM, Riew KD. Cervical disc arthroplasty: tips and tricks. Int Orthop. 2019;43(4):777-783.

5. Wang QL, Tu ZM, Hu P, Kontos F, Li YW, Li L, et al. Long-term results comparing cervical disc arthroplasty to anterior cervical discectomy and fusion: a systematic review and meta-analysis of randomized controlled trials. Orthop Surg. 2020;12(1):16-30.

6. Latka D, Kozlowska K, Miekisiak G, et al. Safety and efficacy of cervical disc arthroplasty in preventing the adjacent segment disease: a meta-analysis of mid- to long-term outcomes in prospective, randomized, controlled multicenter studies. Ther Clin Risk Manag. 2019;15:531-539.

7. Zhang Y, Lv N, He F, Pi B, Liu H, Chen AC, et al. Comparison of cervical disc arthroplasty and anterior cervical discectomy and fusion for the treatment of cervical disc degenerative diseases on the basis of more than 60 months of follow-up: a systematic review and meta-analysis. BMC Neurol. 2020;20(1):143.

8. Health Quality Ontario. Cervical artificial disc replacement versus fusion for cervical degenerative disc disease: a health technology assessment. Ont Health Technol Assess Ser. 2019;19(3):1-223.

9. Boselie TF, Willems PC, van Mameren H, de Bie R, Benzel EC van Santbrink H. WITHDRAWN: Arthroplasty versus fusion in single-level cervical degenerative disc disease. Cochrane Database Syst Rev. 2015;2015(5):CD009173.

10. Vital JM, Guerin P, Gille O, et al. The Mobi-Cervical disc prosthesis: indications, technique and results. Interact Surg. 2008;3:181-186.

11. Montano N, Ricciardi L, Olivi A. Comparison of anterior cervical decompression and fusion versus laminoplasty in the treatment of multilevel cervical spondylotic myelopathy: a meta-analysis of clinical and radiological outcomes. World Neurosurg. 2019;130:530-536.e2.

12. Flynn TB. Neurologic complications of anterior cervical interbody fusion. Spine (Phila Pa 1976). 1982;7(6):536-539.

13. Apfelbaum RI, Kriskovich MD, Haller JR. On the incidence, cause, and prevention of recurrent laryngeal nerve palsies during anterior cervical spine surgery. Spine (Phila Pa 1976). 2000;25(22):2906-2912.

14. Epstein NE. A review of complication rates for anterior cervical diskectomy and fusion (ACDF). Surg Neurol Int. 2019;10:100.

15. Rahimizadeh A, Soufiani H, Rahimizadeh S. Remote cervical pseudomeningocele following anterior cervical corpectomy and fusion: report of a case and review of the literature. Int J Spine Surg. 2016;10:36.

16. Newhouse KE, Lindsey RW, Clark CR, Lieponis J, Murphy MJ. Esophageal perforation following anterior cervical spine surgery. Spine (Phila Pa 1976). 1989;14(10):1051-1053.

17. Halani SH, Baum GR, Riley JP, et al. Esophageal perforation after anterior cervical spine surgery: a systematic review of the literature. J Neurosurg Spine. 2016;25(3):285-291.

18. Yee TJ, Swong K, Park P. Complications of anterior cervical spine surgery: a systematic review of the literature. J Spine Surg. 2020;6(1):302-322.
19. Novegno F, Granaroli P, Ciccoritti L, Lunardi P, Fraioli MF. Chylous fistula: management of a rare complication following right anterior cervical spine approach. Eur Spine J. 2019;28(suppl 2):61-67.

20. Wang XF, Meng Y, Liu H, Hong Y, Wang BY. Anterior bone loss after cervical disc replacement: a systematic review. World J Clin Cases. 2020;8(21):5284-5295.

21. Parish JM, Asher AM, Coric D. Complications and complication avoidance with cervical total disc replacement. Int J Spine Surg. 2020;14(s2):S50-S56.

22. Jain NS, Nguyen A, Formanek B, et al. Cervical disc replacement: trends, costs, and complications. Asian Spine J. 2020;14(5):647-654.

23. Park MS, Lee YB, Moon SH, et al. Facet joint degeneration of the cervical spine: a computed tomographic analysis of 320 patients. Spine (Phila Pa 1976). 2014;39(12):E713-E718.

24. Malham GM, Parker RM, Ellis NJ, Chan PG, Varma D. Cervical artificial disc replacement with ProDisc-C: clinical and radiographic outcomes with long-term follow-up. J Clin Neurosci. 2014;21(6): 949-953.

25. Hou Y, Liu Y, Yuan W, et al. Cervical kinematics and radiological changes after Discover artificial disc replacement versus fusion. Spine J. 2014;14(6):867-877.

26. Tsermoulas G, Bhattathiri PS. Anterior migration of prosthesis following cervical arthroplasty. Br J Neurosurg. 2013;27(1):132-133

27. Pelletier Y, Gille O, Vital JM. An anterior dislocation after Mobi-C cervical disc arthroplasty. Asian J Neurosurg. 2020;15(3): 719-721.

28. Pickett GE, Sekhon LH, Sears WR, Duggal N. Complications with cervical arthroplasty. J Neurosurg Spine. 2006;4(2):98-105.

29. Kunkel ST, Moschetti WE, Werth P, et al. Tobacco exposure is associated with extremely low polyethylene oxidation in total knee arthroplasty components. Arthroplast Today. 2021;8:243-246.

\section{Disclosures}

The authors report no conflict of interest concerning the materials or methods used in this study or the findings specified in this paper.

\section{Author Contributions}

Conception and design: Prod'homme, Boscherini. Acquisition of data: Prod'homme, Grasset. Analysis and interpretation of data: Prod'homme. Drafting the article: Prod'homme. Critically revising the article: Prod'homme, Grasset. Reviewed submitted version of manuscript: Prod'homme, Grasset. Approved the final version of the manuscript on behalf of all authors: Prod'homme. Administrative/technical/material support: Grasset. Study supervision: Boscherini.

\section{Correspondence}

Marc Prod'homme: Neuro Orthopedic Center, Clinic La Source, Lausanne, VD, Switzerland. marcprod86@gmail.com. 